\title{
Welches Sommerloch?
}

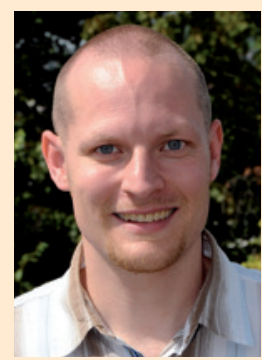

Christian Schäfer, Stuttgart
Ein Sommerloch gab es wirklich nicht in diesem Jahr. Zumindest nicht bei der Dialyse aktuell. Man könnte sich natürlich auch fragen, ob sich die letzten Wochen aufgrund des meist doch eher herbstlichen Wetters überhaupt als echter Sommer bezeichnen lassen. Das Wort „Sommerloch“ wäre damit sowieso ad adsurbum geführt worden bzw. hätte eine andere Bedeutung erlangt - das Loch sozusagen als Fehlen des (Hoch-)Sommers an sich. Aber diese Diskussion ist eine andere Geschichte.

Zurück zur Dialyse aktuell und den aktuellen Entwicklungen: Mit Freude kann ich Ihnen mitteilen, dass wir eine Kooperation mit der Deutschen Gesellschaft für Nephrologie (DGfN) aufgebaut haben. Ab diesem Heft können Sie in jeder Ausgabe der Dialyse aktuell interessante Neuigkeiten aus der Fachgesellschaft lesen. Im ersten Beitrag ab Seite 352 stellt die DGfN die Imagekampagne „Ihre Nieren liegen uns am Herzen“ vor, die sie zusammen mit dem Verband Deutscher Nierenzentren (DN) e.V., dem KfH Kuratorium für Dialyse und Nierentransplantation e.V. und der PHV Patienten-Heimversorgung Gemeinnützige Stiftung durchführt.

Natürlich ging es bei uns in den Sommermonaten auch um Kongressvorbereitungen: Ab September stehen nämlich wie gewohnt Veranstaltungen rund um die Nephrologie und nephrologische Pflege an. So haben wir Ihnen mit dem Kompendium Nephrologie 2014, das zum 6. Kongress für Nephrologie der DGfN (06.-09.09.2014 in Berlin) erscheint, ein informatives Paket geschnürt, in dem Sie den aktuellen Stand des Wissens zu nephrologisch relevanten Themen vorfinden. Ebenso informiert Sie die Zeitung Current Congress über Highlights auf dem DGfN-Kongress.

Auch die Arbeitsgemeinschaft für nephrologisches Personal e.V. (AfnP) beschäftigt sich mit den kommenden Tagungen und kündigt sie auf ihren Fachgesellschaftsseiten an: So finden in diesem Jahr zum ersten Mal die „Dialyse Basic Days“ (26.-27.09.2014 in Fulda) statt. Die AfnP reagiert hiermit auf den sich anbahnenden Umbau der Dialyseteams infolge der sich verändernden Rahmenbedingungen in der Nephrologie. So vermitteln die Referenten in praxisorientierten Vorträgen und Workshops hauptsächlich Grundlagen rund um die Themen Dialyse, Nephrologie und Pflege. Diese Veranstaltung wird sich in Zukunft mit dem AfnP-Symposium in Fulda im Herbst abwechseln. Lesen Sie hierzu den Beitrag auf Seite 338 in dieser Ausgabe.

Sie finden auf Seite 345 ebenfalls eine erste Ankündigung samt vorläufigem Programm zur Nephro Fachtagung Ulm 2015, die ab nächstem Jahr im Februar stattfinden wird. Interessierte können sich schon einmal den 27.-28.02.2015 vormerken - der Tagungsort hat sich allerdings geändert: Die Veranstaltung findet nun im Kornhaus Ulm statt.

Ihnen ans Herz legen kann ich auch die Artikel zum Schwerpunkt dieser Ausgabe der Dialyse aktuell ab Seite 356, der sich um die Peritonealdialyse (PD) dreht. Immer noch wird die PD - ein der Hämodialyse (HD) mindestens gleichwertiges Verfahren - mit etwa 5\% in Deutschland viel zu selten angewandt, auch im internationalen Vergleich. Die Gründe hierfür sind vielfältig. Grundsätzlich gibt es in Deutschland das Problem, dass zu wenig patientenorientiert gedacht wird. So sind eher strukturelle und personelle Gegebenheiten dafür entscheidend, welche Dialyseform Betroffene auswählen können. Prof. Reinhard Brunkhorst, Hannover, sagte hierzu auf der Fachtagung „Zukunft der Dialyse - Herausforderungen für die Versorgung von morgen“ am 01.07.2014 in Berlin laut einer Pressemeldung des IGES Instituts: „Wir benötigen endlich eine abgestimmte Steuerung der Dialyseversorgung, damit die Wahl des Dialyseverfahrens nur nach medizinischen Kriterien und Fragen der Lebensqualität der Patienten erfolgt. Doch davon sind wir weit entfernt." Man benötige hierfür klar definierte Versorgungsziele, eine adäquate Honorierung der einzelnen Dialyseverfahren sowie eine umfassende Ausbildung von Fachärzten und Pflegepersonal. Wie wahr - in diesem Sinne eine erhellende Lektüre dieser Ausgabe der Dialyse aktuell, des Kompendium Nephrologie 2014 und der Zeitung Current Congress. 